
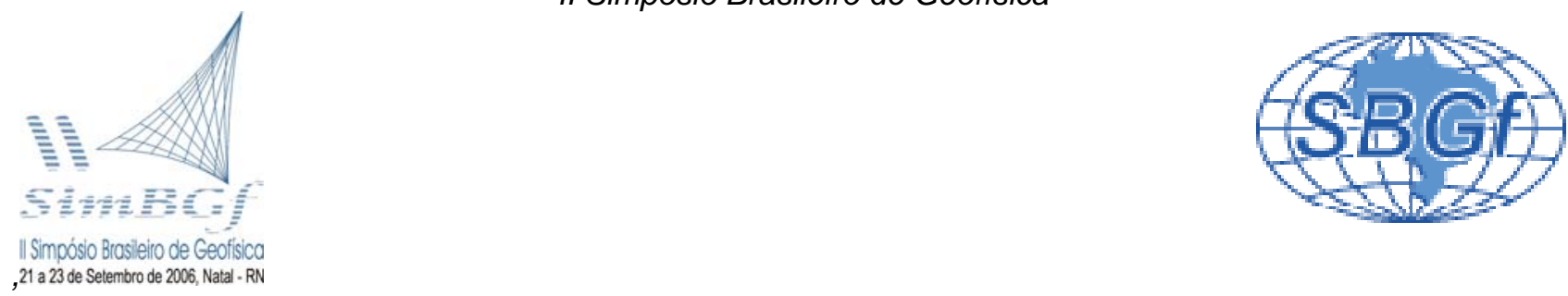

\title{
"Caracterização geológico-ambiental do Lixão de Serrana, na cidade de Ribeirão Preto (SP), com o uso de métodos geoelétricos"
}

Georgia Castro de Souza, Sergio Junior da Silva Fachin \& Vagner Roberto Elis Instituto de Astronomia, Geofísica e Ciências Atmosféricas

Universidade de São Paulo (IAG-USP)

Copyright 2004, SBGf - Sociedade Brasileira de Geofísica

Este texto foi preparado para a apresentação no I Simpósio de Geofísica da Sociedade Brasileira de Geofísica, São Paulo, 26-28 de setembro de 2004. Seu conteúdo foi revisado pela Comissão Tecno-científica do I SR-SBGf mas não necessariamente representa a opinião da SBGf ou de seus associados. E proibida a reprodução total ou parcial deste material para propósitos comerciais sem prévia autorização da SBGf.

\section{Resumo}

A Geofísica aplicada ao estudo de caracterização de áreas contaminadas vem se mostrando de grande importância, pois fornece informações quanto à existência de contaminantes em águas subterrâneas e solos. Este projeto visa avaliar e caracterizar o problema ambiental gerado na região do Lixão de Serrana, que se localiza na cidade de Ribeirão Preto (SP), local em que na década de 1970 houve a disposição inadequada de um grande volume de resíduos domésticos, industriais e hospitalares. O lixão atualmente encontra-se desativado, no entanto, há vestígios dos danos causados na época. Neste projeto foram coletados dados geofísicos da região, que foram analisados e interpretados para obtenção de informações sobre a forma e o comportamento da pluma de contaminação.

\section{Introdução}

O crescimento urbano e industrial gera um aumento dos problemas ambientais, que por sua vez, estimula a busca de métodos de análise do comportamento e possibilidades de preservação do meio ambiente. Esse quadro aponta para a necessidade do aprimoramento das técnicas de investigação existentes e a utilização de novas ferramentas, que possibilitem análises rápidas e eficientes. Dentro deste contexto, os métodos geofísicos têm sido extensamente aplicados, especialmente os métodos da eletrorresistividade e eletromagnético indutivo, que possibilitam a investigação de uma série de características do meio físico, frente ao processo de contaminação de uma maneira rápida e a baixos custos.

Neste estudo são utilizados métodos geofísicos para avaliar e caracterizar o problema ambiental gerado na região do Lixão de Serrana, que se localiza na cidade de Ribeirão Preto - SP.

Neste local, devido à existência de duas cavidades alongadas de cerca de $300 \mathrm{~m}$ de comprimento por 40 a 60 $\mathrm{m}$ de largura, e aproximadamente 12 de profundidade, originadas da extração de areia para a construção civil, iniciou-se em meados da década de 1970 a disposição de resíduos domésticos, industriais e hospitalares, no total foram depositados cerca de $600.000 \mathrm{~m}^{3}$ de resíduos.

Atualmente o depósito se encontra saturado e sem atividade. No entanto, os danos causados pela disposição inadequada dos resíduos ainda estão presentes e a pluma de contaminação gerada ainda se mantém em desenvolvimento.

O lixão encontra-se na área de geologia complexa, com afloramento de arenitos e diabásios mesozóicos, em uma área de recarga do aqüífero Botucatu/Pirambóia. Resultados de trabalhos anteriores no local sugerem dois sentidos de fluxo de águas subterrâneas, para NE e NW (Elis, 1999). Esse comportamento bidimensional do fluxo deve-se ao fato da área ser um divisor topográfico.

Dessa forma, este trabalho visa além da identificação e mapeamento da área afetada pela contaminação, uma caracterização mais detalhada das relações entre as unidades litológicas no local. Para isto, são utilizados os métodos eletromagnéticos indutivo e sondagem elétrica vertical que auxiliam na investigação geológico-ambiental da área.

\section{Metodologia/ Problema Investigado}

As metodologias utilizadas foram o Método Eletromagnético Indutivo e o Método da Eletrorresistividade (SEV), com o objetivo de identificar e mapear as áreas afetadas pelos resíduos e seus contaminantes, bem como contribuir para um melhor conhecimento da geologia de subsuperfície.

\section{Eletromagnético indutivo}

O método eletromagnético indutivo caracteriza-se pelo uso de equipamentos de operações muito simples, rápidas e de baixo custo, fatores que explicam sua extensa aplicação em estudos ambientais.

Os equipamentos utilizados medem a condutividade aparente do meio e são compostos por duas bobinas (emissão e recepção). A bobina transmissora emite um campo magnético primário $H p$, que induz, em subsuperfície, correntes elétricas, que geram um campo secundário Hs. A combinação destes dois campos é medida pela bobina receptora, sob certas condições. Dessa forma, a condutividade aparente do meio pode ser obtida por meio da equação (McNeil, 1980):

$$
\sigma_{a}=\frac{4}{\omega \mu_{0} s^{2}}\left[\frac{H_{s}}{H_{p}}\right]
$$


Onde:

$\sigma_{\mathrm{a}}=\mathrm{a}$ condutividade aparente $(\mathrm{mSm} / \mathrm{m})$

$\omega=2 \pi \mathrm{f}$, sendo $\mathrm{f}(\mathrm{Hz})$

$\mu_{0}=$ permeabilidade magnética no vácuo

$\mathrm{s}=$ espaçamento entre as bobinas $(\mathrm{m})$

A partir do equipamento utilizado as profundidades investigadas variam dependendo do comprimento do cabo de referência e da orientação das bobinas. Neste estudo foram adotados espaçamentos entre as bobinas de $10 \mathrm{~m}$, $20 \mathrm{~m}, 40 \mathrm{~m}$, com as bobinas nas posições horizontal e vertical. Com a utilização nessas configurações as profundidades de investigação atingidas foram 7,5 m, 15 $\mathrm{m}, 30 \mathrm{~m}$ e $60 \mathrm{~m}$.

Os dados de condutividade aparente obtidos foram plotados em perfis de condutividade aparente, em função da distância, e a partir do conjunto destes perfis foi possível à elaboração de mapas de variação de condutividade aparente para as várias profundidades investigadas.

A interpretação desses dados é qualitativa, porém existem, em desenvolvimento, softwares para quantificação desses dados.

\section{Eletrorresistividade}

O método da eletrorresistividade utiliza uma corrente artificial que é introduzida no terreno através de dois eletrodos (denominados de A e B), com o objetivo de medir o potencial gerado em outros dois eletrodos (denominados de $\mathrm{M}$ e $\mathrm{N}$ ) nas proximidades do fluxo de corrente, permitindo assim calcular a resistividade real ou aparente em subsuperfície. Para esse dispositivo de quatro eletrodos a resistividade é assumida como constante e pode ser calculada por:

Onde:

$$
\rho=K . \frac{\Delta V}{I}
$$

$\rho=$ resistividade elétrica (ohm. $\mathrm{m}$ )

$\mathrm{K}=$ fator geométrico

$\Delta \mathrm{V}=$ diferença de potencial entre os eletrodos $\mathrm{M}$ e $\mathrm{N}$

$\mathrm{I}=$ intensidade de corrente que passa entre os eletrodos $\mathrm{A}$ e B

$\mathrm{O}$ fator $\mathrm{K}$ depende da geometria do arranjo de eletrodos na superfície e pode ser calculado através da fórmula geral:

$$
K=\frac{2 \pi}{(1 / A M)-(1 / A N)-(1 / B M)+(1 / B N)}
$$

A técnica de sondagem elétrica vertical consiste, basicamente, na análise e interpretação da variação de resistividade com a profundidade de um parâmetro físico, obtido a partir de medidas efetuadas na superfície do terreno. $\mathrm{O}$ arranjo de campo utilizado para os ensaios de sondagem elétrica vertical, neste trabalho, foi 0 denominado Schlumberger, em que os eletrodos dispostos no terreno estão de acordo com um mesmo alinhamento. O ponto de atribuição do ensaio é o centro geométrico do arranjo. A principal característica desse arranjo é que à distância $M N$ que deve ser bastante pequena em relação à $A B$, procurando sempre satisfazer a relação $M N \leq A B / 5$.
Como os valores de resistividade calculados refletem na influência dos vários estratos investigados, estas são denominadas resistividades aparentes. Os valores de resistividade aparente obtidos são plotados em relação aos valores da distância $A B / 2$, fornecendo a curva de resistividade aparente, a qual é passível de interpretação quantitativa por meio do uso de softwares apropriados (Moscou University, 2004). O processo consiste em interpretar as sondagens levando em consideração um modelo geoelétrico isotrópico e homogêneo, onde a resistividade varia somente com a profundidade. $\mathrm{Na}$ interpretação, admite-se um modelo inicial e calcula-se a curva teórica correspondente, com o auxílio do programa computacional, comparando-se com a curva de campo, procurando criar um modelo mais confiável, modificando os valores dos parâmetros.

\section{Resultados}

No local foram realizadas 13 linhas de caminhamento eletromagnético e 2 sondagens elétricas verticais (SEV). A Figura 1 apresenta as localizações das linhas de EM e das duas SEV's realizadas no local, que representam os dados discutidos neste trabalho.

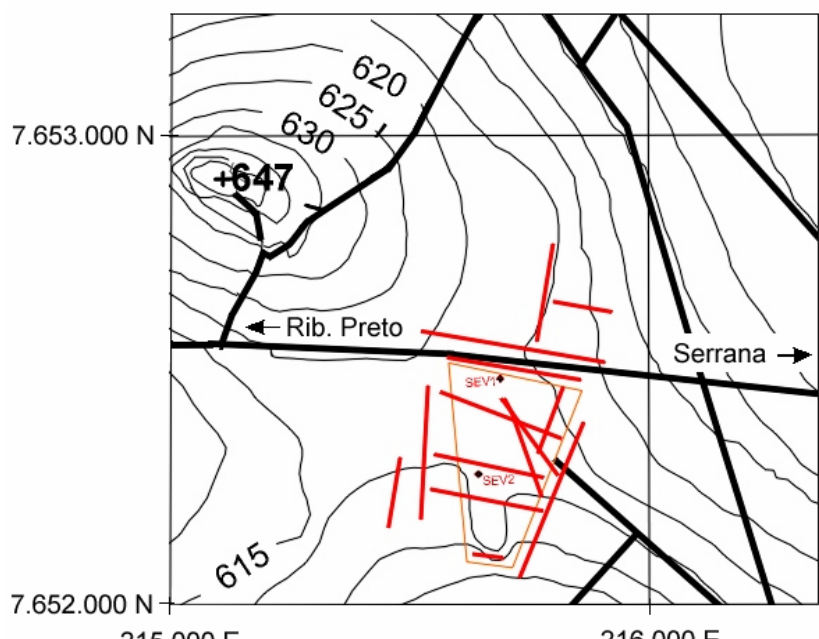

$215.000 \mathrm{E}$

216.000 E

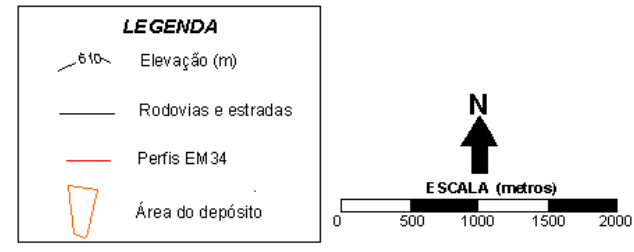

Figura 1: Mapa de localização da área e posição das linhas de EM34 e das SEV's.

Sondagens elétricas verticais (SEV's)

A partir dos dados das curvas de sondagens e de informações sobre a geologia local foi possível estudar o perfil geológico sobre as cavas (SEV-02) e fora destas (SEV-01). Estas informações estão ilustradas nas Figuras 2 e 3 e nas Tabelas 1 e 2. 


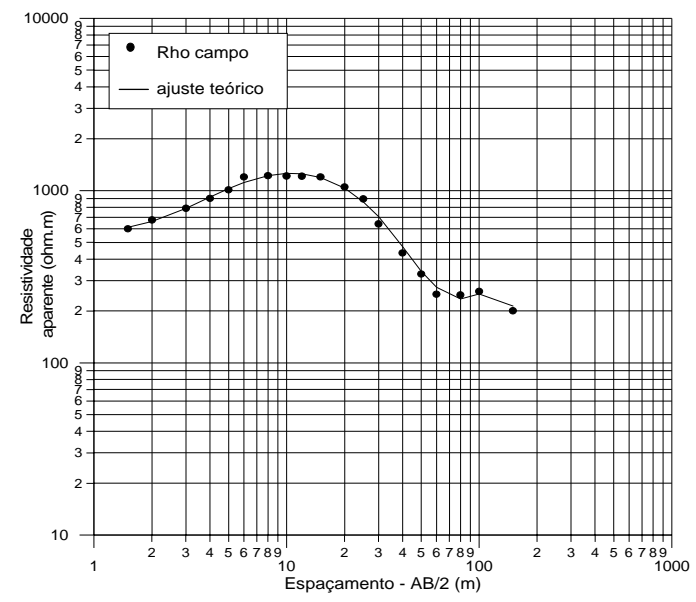

Figura 2: SEV-01 (Região fora das cavas de resíduos)

\begin{tabular}{|c|c|c|c|}
\hline Camadas & Rho (ohm.m) & $\begin{array}{c}\text { Espessura } \\
(\mathrm{m})\end{array}$ & $\begin{array}{c}\text { Perfil } \\
\text { sugerido }\end{array}$ \\
\hline 1 & 564,0 & 1,7 & \multirow{3}{*}{$\begin{array}{c}\text { Colúvio } \\
+ \\
\text { Solo }\end{array}$} \\
\hline 2 & 2682,0 & 3,2 & \\
\hline 3 & 885,0 & 4,5 & \\
\hline 4 & 70,0 & 15,0 & $\begin{array}{c}\text { Zona } \\
\text { Saturada }\end{array}$ \\
\hline 5 & 1833,0 & 13,3 & Basalto \\
\hline 6 & 22,0 & ----- & Arenito \\
\hline
\end{tabular}

Tabela 1: Perfil geoelétrico da SEV-01

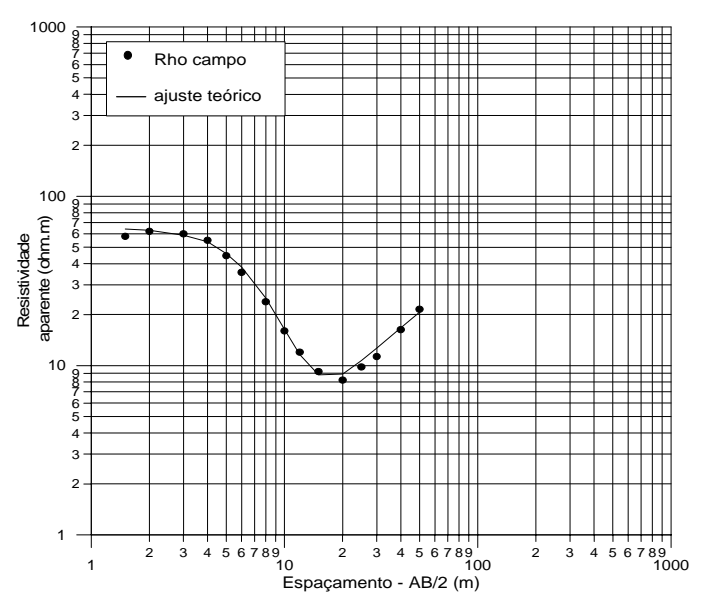

Figura 3: SEV-02 (Região sobre as cavas de resíduos)

\begin{tabular}{|c|c|c|c|}
\hline Camadas & Rho (ohm.m) & $\begin{array}{c}\text { Espessura } \\
\text { (m) }\end{array}$ & $\begin{array}{l}\text { Perfil } \\
\text { sugerido }\end{array}$ \\
\hline 1 & 64,0 & 1,9 & \multirow{2}{*}{$\begin{array}{l}\text { Cobertura } \\
\text { do aterro }\end{array}$} \\
\hline 2 & 83,0 & 1.3 & \\
\hline 3 & 2,7 & 6.1 & Resíduos \\
\hline 4 & 346,0 & $\begin{array}{l}---- \\
\end{array}$ & Arenito \\
\hline
\end{tabular}

Tabela 2: Perfil geoelétrico da SEV-02
$\mathrm{Na}$ área fora dos resíduos são individualizadas três unidades: a primeira unidade, superficial, caracterizada por sedimentos coluvionares. Uma outra unidade é representada por basaltos e os solos de alteração deste tipo de rocha, e por fim ocorrem os arenitos da Formação Botucatu que encontram-se recobertos pelos basaltos em algumas partes da área. Na SEV-01 pode-se verificar essa situação, onde uma camada de alta resistividade ocorre sobre o arenito, que é característica do basalto presente na área. Esse modelo é corroborado por informações diretas (poços de monitoramento existentes no local). Ainda de acordo com informações do poço e dados geofísicos, na maior parte da região sob as cavas o colúvio recobre diretamente 0 arenito Botucatu, sem a existência do basalto. Esse é o modelo obtido para a SEV-02, onde os resíduos foram dispostos em uma cava construída no colúvio e em contato direto com os arenitos não saturados.

\section{Caminhamentos eletromagnéticos}

Foram realizados 13 caminhamentos eletromagnéticos, no entanto, neste texto serão apresentadas apenas 2 pseudo-seções, de forma a caracterizar a área sobre os resíduos; região das cavas, e a área sem a presença destes, mas afetada pelos contaminantes a jusante; localizada fora das cavas, em suas margens.

$\mathrm{Na}$ pseudo-seção da linha 1 (Figura 4) localizada a norte (N) das cavas, foram verificados valores de baixa condutividade aparente (alta resistividade aparente) na parte superficial da seção, no entanto valores mais elevados de condutividade aparente nas porções em maiores profundidades, que pode caracterizar o deslocamento da pluma de contaminação.

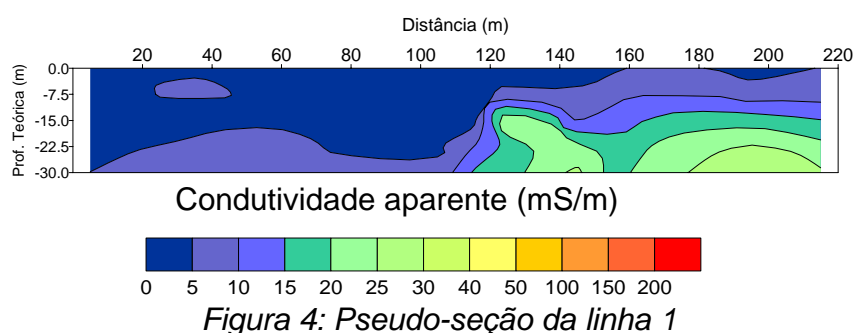

$\mathrm{Na}$ pseudo-seção da linha 4 (Figura 5) executada transversalmente as cavas, aproximadamente no centro destas, foi possível evidenciar a localização dos resíduos, caracterizando a posição das duas cavas, local onde foram encontrados os valores mais altos de condutividade aparente.

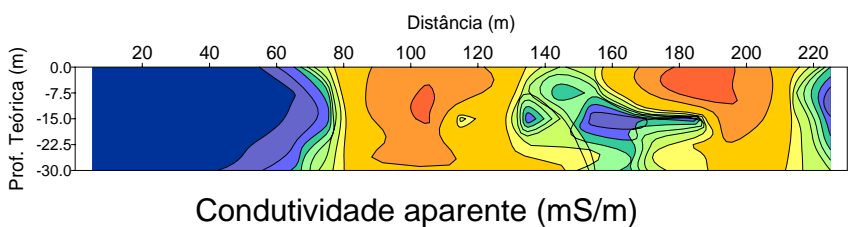

Condutividade aparente $(\mathrm{mS} / \mathrm{m})$

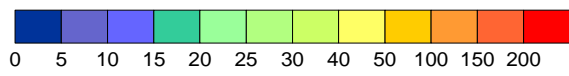

Figura 5: Pseudo-seção da linha 4 
Após serem analisadas todas as pseudo-seções foram gerados mapas a partir de todos os dados. Estes mostram o comportamento da possível pluma de contaminação nas profundidades teóricas de 7,5 m e $30 \mathrm{~m}$ (Figuras 6 e 7).

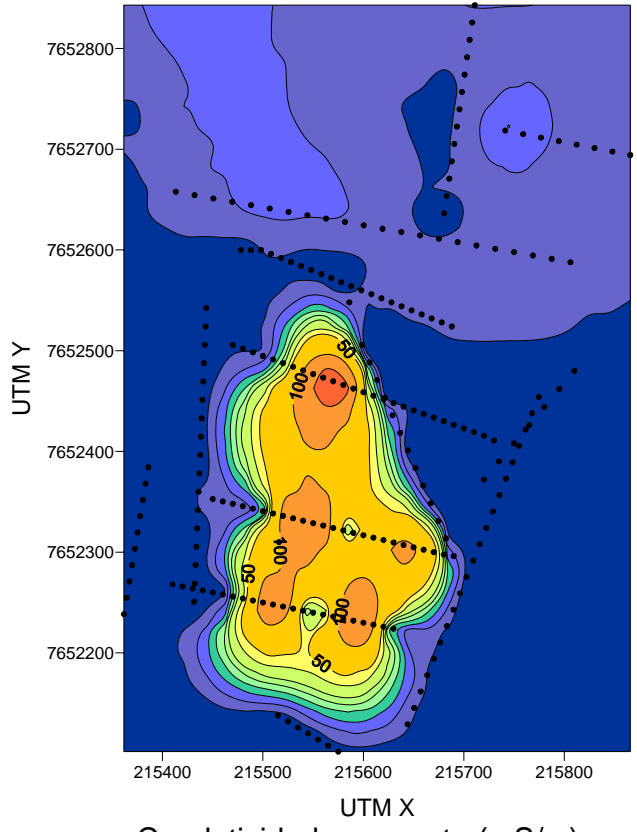

Condutividade aparente $(\mathrm{mS} / \mathrm{m})$

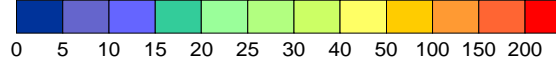

Figura 6: Mapa de condutividade $(\mathrm{mS} / \mathrm{m})$ da área toda do Lixão de Serrana, profundidade de investigação de 7,5 m.

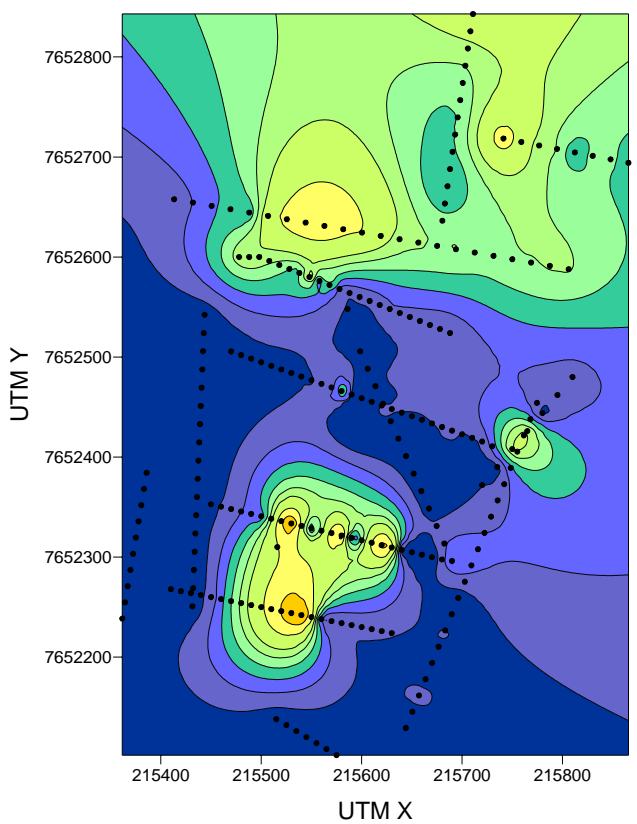

Condutividade aparente $(\mathrm{mS} / \mathrm{m})$

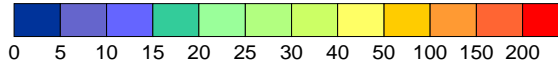

Figura 7: Mapa de condutividade $(\mathrm{mS} / \mathrm{m})$ da área toda do Lixão de Serrana, profundidade de investigação de $30 \mathrm{~m}$.
A partir destes mapas foi possível detalhar a posição de forma mais precisa da área de abrangência da pluma de contaminação gerada pelos resíduos, no Lixão de Serrana. Verificou-se que mais superficialmente, na profundidade de $7,5 \mathrm{~m}$ a contaminação é bastante acentuada, apresentando altos valores de condutividade aparente, em torno de $150 \mathrm{mS} / \mathrm{m}$ e maiores dimensões. Já na profundidade teórica de $30 \mathrm{~m}$, abaixo das cavas, mas em posição de ocorrência da zona saturada, de acordo com informações de alguns poços de monitoramento, a zona anômala apresenta valores de condutividade aparente não tão elevados, em torno de $25 \mathrm{mS} / \mathrm{m}$, e dimensões reduzidas no local das cavas. No entanto há prolongamentos de zonas de condutividades médias a altas (acima de $30 \mathrm{mS} / \mathrm{m}$ ) ao norte $(\mathrm{N})$, sugerindo um espalhamento dos contaminantes em profundidade, afetando a zona saturada.

\section{Discussão e Conclusões}

A partir das informações obtidas e da análise do local verifica-se que a área atualmente ainda apresenta altos índices de contaminação, principalmente nos locais das cavas e na porção superficial do terreno. No entanto também há presença de um deslocamento da pluma de contaminação, principalmente na direção norte $(\mathrm{N})$.

Com a associação dos dados elétricos e eletromagnéticos foi possível se obter uma boa caracterização geológicoambiental da área, com informações quanto à geologia local, a partir dos dados das sondagens elétricas verticais (SEV's) e quanto ao comportamento da contaminação, a partir dos dados dos caminhamentos eletromagnéticos (pseudo-seções).

É importante lembrar que a integração dos dados geofísicos aos dados diretos dos poços de monitoramento existentes no local, foram importantes para ajustar e refinar os modelos geológico-ambientais obtidos.

\section{Agradecimentos}

Agradecemos à FAPESP pelo auxílio-pesquisa 99/12216-9 e a estrutura do IAG/USP para a realização do projeto de pesquisa.

\section{Referências Bibliográficas}

Elis, V.R., 1999. Avaliação da aplicabilidade de métodos elétricos de prospecção geofísica no estudo de áreas utilizadas para disposição de resíduos. Tese de Doutorado, Instituto de Geociências e Ciências Exatas, UNESP, Campus de Rio Claro-SP, 264 p.

McNell, J.D., 1980. Eletromagnetic terrain conductivity measurement at low induction numbers. Nota técnica $n^{0} 6$, Geonics, 15p.

Moscou University, 2004. IPI-1D. Minimal (Free) programs for 1D VES data interpretation. The laboratory of nearsurface electrical prospecting, Moscou University. 